Research Paper

\title{
Type and Location of Placenta Previa Affect Preterm Delivery Risk Related to Antepartum Hemorrhage
}

\author{
Atsuko Sekiguchi ${ }^{凶}$, Akihito Nakai, Ikuno Kawabata, Masako Hayashi, Toshiyuki Takeshita \\ Department of Obstetrics and Gynecology, Nippon Medical School, Tokyo, Japan. \\ $\triangle$ Corresponding author: Atsuko Sekiguchi Tel: 81-(0)42 371 2111, Fax: 81-(0)42 372 7372, E-mail: oya-a@nms.ac.jp. \\ (c) Ivyspring International Publisher. This is an open-access article distributed under the terms of the Creative Commons License (http://creativecommons.org/ \\ licenses/by-nc-nd/3.0/). Reproduction is permitted for personal, noncommercial use, provided that the article is in whole, unmodified, and properly cited.
}

Received: 2013.04.06; Accepted: 2013.09.15; Published: 2013.09.24

\begin{abstract}
Purpose: To evaluate whether type and location of placenta previa affect risk of antepartum hemorrhage-related preterm delivery. Methods: We retrospectively studied 162 women with singleton pregnancies presenting placenta previa. Through observation using transvaginal ultrasound the women were categorized into complete or incomplete placenta previa, and then assigned to anterior and posterior groups. Complete placenta previa was defined as a placenta that completely covered the internal cervical os, with the placental margin $>2 \mathrm{~cm}$ from the os. Incomplete placenta previa comprised marginal placenta previa whose margin adjacent to the internal os and partial placenta previa which covered the os but the margin situated within $2 \mathrm{~cm}$ of the os. Maternal characteristics and perinatal outcomes in complete and incomplete placenta previa were compared, and the differences between the anterior and the posterior groups were evaluated. Results: Antepartum hemorrhage was more prevalent in women with complete placenta previa than in those with incomplete placenta previa $(59.1 \%$ versus $17.6 \%)$, resulting in the higher incidence of preterm delivery in women with complete than in those with incomplete placenta previa [ $45.1 \%$ versus $8.8 \%$; odds ratio (OR) 8.51 ; $95 \%$ confidence interval $(\mathrm{Cl}) 3.59-20.18$; $\mathrm{p}<0.00 \mathrm{I}$ ]. In complete placenta previa, incidence of antepartum hemorrhage did not significantly differ between the anterior and the posterior groups. However, gestational age at bleeding onset was lower in the anterior group than in the posterior group, and the incidence of preterm delivery was higher in the anterior group than in the posterior group $(76.2 \%$ versus $32.0 \%$; OR $6.8 ; 95 \% \mathrm{Cl}$ 2.12-21.84; $p=0.002$ ). In incomplete placenta previa, gestational age at delivery did not significantly differ between the anterior and posterior groups. Conclusion: Obstetricians should be aware of the increased risk of preterm delivery related to antepartum hemorrhage in women with complete placenta previa, particularly when the placenta is located on the anterior wall.
\end{abstract}

Key words: complete placenta previa, anterior placenta previa, preterm delivery, antepartum hemorrhage.

\section{Introduction}

The prevalence of placenta previa has been recently estimated to be approximately $0.5 \%$ of all pregnancies, and this increase correlates to the elevated cesarean section rate ${ }^{1}$. Placenta previa is a major cause of maternal morbidity and mortality because of the associated massive antepartum and intrapartum hemorrhage 2,3 . Moreover, placenta previa is associated with preterm delivery, with the neonatal mortality increasing threefold as a result of prema- turity ${ }^{4}$. Although placenta previa is associated with antepartum hemorrhage, massive hemorrhage necessitating preterm cesarean section is not observed in all women with the condition. The ability to predict severe antepartum hemorrhage and emergency cesarean section is critical in the management of placenta previa.

To date, no consensus exists on the preterm delivery risk of different types and locations of placenta 
previa. Only a few reports have focused on the maternal and perinatal outcomes of different types of placenta previa $5,6,7,8$. Furthermore, the effect of anterior/posterior placental position on preterm delivery is unknown, although increased perinatal risks, including placenta accreta, excessive intraoperative blood loss, hysterectomy, and neonatal anemia, have been reported to be associated with anterior placenta previa 9,10 .

In the present study, we evaluated whether different types and locations of placenta previa influence risk of antepartum hemorrhage-related preterm delivery.

\section{Methods}

We retrospectively analyzed the medical records of 164 women with singleton pregnancies presenting placenta previa whose deliveries were conducted at our institute between January 2004 and March 2012. Data were obtained during routine care at our institution. Informed consent was obtained from each patient and protection of personal data and confidentiality were prioritized. Institutional review board approval was obtained, and the study has been performed in accordance with the ethical standards laid down in the 1964 Declaration of Helsinki and its later amendments.

Two women whose indications for preterm cesarean section were not antepartum hemorrhage were excluded, and a total of 162 women were finally included in this study. Patients were categorized into complete or incomplete placenta previa according to the type of placenta previa, and they were assigned to anterior and posterior groups according to placental location. Maternal characteristics, and perinatal outcomes, including admission, tocolytic use, antepartum hemorrhage, gestational age at bleeding onset, gestational age at delivery, birth weight, Apgar score, umbilical artery $\mathrm{pH}$, placenta accreta incidence, anterior placental position, cervical length at delivery, and intraoperative blood loss, were compared between women with complete and incomplete placenta previa. In addition, differences between the anterior and posterior groups were evaluated.

According to our hospital protocol, asymptomatic women with placenta previa were treated as outpatients. However, if bleeding or frequent uterine contractions were observed, patients were immediately admitted to the hospital, where treatment, including bed rest, vaginal lavage, and augmentation of tocolytic agents such as ritodrine, magnesium sulfate, and progesterone, was implemented. Scheduled elective cesarean section for placenta previa was usually performed at 37 weeks of gestation according to our institutional protocol, but was occasionally performed early in the 38th week in stable cases. Preterm cesarean section was performed only when massive, uncontrollable hemorrhage occurred. Blood loss over approximately $200 \mathrm{ml}$ and continuous hemorrhage without tendency of decrease is the indication for emergent cesarean section in our institute. In women with placenta accreta, cesarean hysterectomy was performed concurrently.

In all the subjects the diagnosis of placenta previa was confirmed by transvaginal ultrasound, performed by trained attending physicians within 1 week of cesarean section after placental migration. Complete placenta previa was defined as a placenta that completely covered the internal cervical os, with the placental margin $>2 \mathrm{~cm}$ from the os. Incomplete placenta previa comprised partial and marginal placenta previa ${ }^{6}$. Partial placenta previa was defined as when the placenta partially covered, but the placental margin was situated within $2 \mathrm{~cm}$ of the internal os. Marginal placenta previa was defined when the placental margin was situated adjacent to the internal os, with the placenta not covering the os.

We employed this classification of complete and incomplete placenta previa because precise differential diagnosis of partial and marginal placenta previa is reported to be sometimes difficult in the absence of cervical dilatation ${ }^{11}$. Women with low-lying placenta were excluded because their clinical management differed. Placental location was categorized as anterior or posterior, on the basis of the side of the uterine wall to which placenta was attached. Placenta accreta was diagnosed only when direct invasion of trophoblast cells into the myometrium was histologically confirmed after hysterectomy.

Statistical analysis was performed using StatMate III (ATMS Co. Ltd., Tokyo, Japan). For categorical variables, the chi-square test or Fisher's exact test was applied. For continuous variables, depending on their distribution, the independent $t$-test or nonparametric Mann-Whitney $U$ test was used. A p-value $<0.05$ was considered statistically significant.

\section{Results}

Of the 162 women included in this study, 71 $(43.8 \%)$ had complete placenta previa and 91 (56.2\%) had incomplete placenta previa. Thirty-one women (19.1\%) had anterior and 131 (80.9\%) had posterior placental position. There were no significant differences in maternal characteristics between women with complete and incomplete placenta previa, except in the prior cesarean rate, which was higher in women with complete placenta previa than in those with incomplete placenta previa [odds ratio (OR) 3.18; 95\% confidence interval (CI) 1.14-8.84; $\mathrm{p}=0.04$; Table 1].

Perinatal outcomes in women with complete and 
incomplete placenta previa are shown in Table 2. Antepartum hemorrhage was more prevalent in women with complete placenta previa than in those with incomplete placenta previa $(59.1 \%$ versus $17.6 \%$; OR 6.79; 95\% CI 3.31-13.92; p < 0.001). Consequently, the incidence of preterm delivery was higher in women with complete placenta previa than in those with incomplete placenta previa ( $45.1 \%$ versus $8.8 \%$; OR 8.51 ; 95\% CI 3.59-20.18; $\mathrm{p}<0.001$ ), with a higher incidence of delivery before 34 weeks of gestation in complete placenta previa $(18.3 \%$ versus $1.1 \%$; OR $24.38 ; 95 \%$ CI 3.09-192; $p<0.001)$. The incidences of birth weight $<2500 \mathrm{~g}$ and $<2000 \mathrm{~g}$ both increased in women with complete placenta previa ( $<2500$ g: OR $2.64 ; 95 \%$ CI
1.31-5.33; $\mathrm{p}<0.01$, and <2000 g: OR 5.97; 95\% CI $1.61-22.06 ; p<0.007)$. However, there were no significant differences in the incidence of Apgar scores $<7$ at 1 and $5 \mathrm{~min}$ and umbilical arterial $\mathrm{pH}$ between women with complete and incomplete placenta previa. Placenta accreta and anterior placental position were significantly more prevalent in women with complete placenta previa than in those with incomplete placenta previa, and intraoperative blood loss was increased in women with complete placenta previa. The prevalence of short cervical length $(\leq 35 \mathrm{~mm})$ did not significantly differ between women with complete and incomplete placenta previa.

Table I. Maternal characteristics in complete and incomplete placenta previa.

\begin{tabular}{|c|c|c|c|c|c|c|}
\hline & Total & Complete & Incomplete & OR & $(95 \% \mathrm{CI})$ & P value \\
\hline & $\mathrm{n}=162$ & $\mathrm{n}=71$ & $\mathrm{n}=91$ & & & \\
\hline Maternal age at delivery (years) & $33.5 \pm 4.4$ & $33.5 \pm 4.4$ & $33.4 \pm 4.4$ & & & 0.886 \\
\hline Nulligravida & $47(29.0 \%)$ & $23(32.4 \%)$ & $24(26.4 \%)$ & 1.34 & $(0.68-2.64)$ & 0.507 \\
\hline Nullipara & $68(42.0 \%)$ & $29(40.8 \%)$ & $39(42.9 \%)$ & 0.92 & $(0.49-1.73)$ & 0.923 \\
\hline Prior preterm delivery & $6(3.7 \%)$ & $4(5.6 \%)$ & $2(2.2 \%)$ & 2.66 & $(0.47-14.94)$ & 0.466 \\
\hline Prior cesarean delivery & $19(11.7 \%)$ & $13(18.3 \%)$ & $6(6.6 \%)$ & 3.18 & $(1.14-8.84)$ & 0.04 \\
\hline Prior dilatation and curettage & $58(35.8 \%)$ & $24(33.8 \%)$ & $34(37.4 \%)$ & 0.86 & $(0.45-1.64)$ & 0.761 \\
\hline ART & $13(8.0 \%)$ & $7(9.9 \%)$ & $6(6.6 \%)$ & 1.55 & $(0.50-4.83)$ & 0.64 \\
\hline
\end{tabular}

Data are expressed as mean $\pm \mathrm{SD}$ or number (\%). OR, odds ratio. CI, confidence interval.

Table 2. Perinatal outcomes in complete and incomplete placenta previa.

\begin{tabular}{|c|c|c|c|c|c|c|}
\hline & Total & Complete & Incomplete & OR & $(95 \% \mathrm{CI})$ & P value \\
\hline & $\mathrm{n}=162$ & $\mathrm{n}=71$ & $\mathrm{n}=91$ & & & \\
\hline Admission & $70(43.2 \%)$ & $44(62.0 \%)$ & $26(28.6 \%)$ & 4.07 & $(2.10-7.89)$ & $<0.001$ \\
\hline Use of tocolytics & $43(26.5 \%)$ & $31(43.7 \%)$ & $12(13.2 \%)$ & 5.1 & $(2.37-10.99)$ & $<0.001$ \\
\hline Antepartum bleeding & $58(35.8 \%)$ & $42(59.1 \%)$ & $16(17.6 \%)$ & 6.79 & $(3.31-13.92)$ & $<0.001$ \\
\hline Gestational age at bleeding onset (weeks) & $30.1(17.1-38.4)$ & $30.1(17.1-36.7)$ & $30.9(25.4-35.1)$ & & & 0.531 \\
\hline Gestational age at delivery (weeks) & $37.4(25.6-38.4)$ & $37.0(25.6-38.4)$ & $37.6(33.9-38.4)$ & & & $<0.001$ \\
\hline$<34$ weeks & $14(8.6 \%)$ & $13(18.3 \%)$ & $1(1.1 \%)$ & 24.38 & $(3.09-192.00)$ & $<0.001$ \\
\hline$<37$ weeks & $40(24.7 \%)$ & $32(45.1 \%)$ & $8(8.8 \%)$ & 8.51 & $(3.59-20.18)$ & $<0.001$ \\
\hline Birth weight $(\mathrm{g})$ & $2673(610-3740)$ & $2608(610-3740)$ & $2768(1958-3432)$ & & & $<0.001$ \\
\hline$<2000 \mathrm{~g}$ & $15(9.3 \%)$ & $12(16.9 \%)$ & $3(3.3 \%)$ & 5.97 & $(1.61-22.06)$ & 0.007 \\
\hline$<2500 \mathrm{~g}$ & $46(28.4 \%)$ & $28(39.4 \%)$ & $18(19.8 \%)$ & 2.64 & $(1.31-5.33)$ & $<0.01$ \\
\hline Apgar Score $1 \mathrm{~min}<7$ & $11(6.8 \%)$ & $8(11.3 \%)$ & $3(3.3 \%)$ & 3.72 & $(0.95-14.60)$ & 0.092 \\
\hline Apgar Score $5 \mathrm{~min}<7$ & $3(1.9 \%)$ & $3(4.2 \%)$ & $0(0.0 \%)$ & & NA & 0.164 \\
\hline Umbilical arterial $\mathrm{pH}$ & $7.319(7.018-7.493)$ & $7.328(7.018-7.493)$ & $7.317(7.057-7.425)$ & & & 0.239 \\
\hline Placenta accreta & $10(6.2 \%)$ & $8(11.3 \%)$ & $2(2.2 \%)$ & 5.65 & $(1.16-27.51)$ & 0.04 \\
\hline Anterior position of placenta & $31(19.1 \%)$ & $21(29.6 \%)$ & $10(11.0 \%)$ & 3.4 & $(1.48-7.81)$ & 0.005 \\
\hline Cervical length at delivery $\leq 35 \mathrm{~mm}$ & $69(42.6 \%)$ & $33(46.5 \%)$ & $36(39.6 \%)$ & 1.33 & $(0.71-2.49)$ & 0.469 \\
\hline Blood loss during operation (ml) & $1260(350-12870)$ & $1435(360-12870)$ & $1110(350-6120)$ & & & $<0.001$ \\
\hline
\end{tabular}

Data are expressed as number (\%) or median (range). OR, odds ratio. CI, confidence interval. NA, Not applicable. 
No significant differences were evident in maternal characteristics and treatments such as admission and tocolytic use, between the anterior and the posterior groups among women with complete placenta previa (Tables 3 and 4). In complete placenta previa, incidence of antepartum hemorrhage did not significantly differ between the anterior and the posterior groups $(76.2 \%$ versus $54.0 \%$; OR 2.73 ; $95 \% \mathrm{CI}$ $0.86-8.59, \mathrm{p}=0.139)$. However, median gestational age at bleeding onset in the anterior group was lower than in the posterior group (26.4 weeks versus 31.4 weeks, $p=0.016)$. The incidence of preterm delivery was higher in the anterior group than in the posterior group $(76.2 \%$ versus $32.0 \%$; OR 6.8 ; $95 \%$ CI $2.12-21.84$; $\mathrm{p}=0.002)$, with a higher incidence of preterm delivery before 34 weeks gestation (38.1\% versus $10.0 \%$; OR
5.54; 95\% CI 1.55-19.85; $p=0.014)$. The incidences of birth weight $<2500 \mathrm{~g}$ and $<2000 \mathrm{~g}$ were both higher in the anterior group than in the posterior group $(<2500$ g: OR 3.79; 95\% CI 1.30-11.04; $\mathrm{p}=0.025$, and $<2000 \mathrm{~g}$; OR 7.08; 95\% CI 1.84-27.27; $\mathrm{p}=0.006$ ). There were no significant differences in umbilical arterial $\mathrm{pH}$ or the incidence of Apgar scores $<7$ at 1 and 5 min between the anterior and posterior groups. The incidence of placenta accreta was higher in the anterior group than in the posterior group (OR 9.6; 95\% CI 1.75-52.66; $\mathrm{p}=$ $0.01)$, resulting in increased intraoperative blood loss.

However, in women with incomplete placenta previa, there were no differences in maternal characteristics and perinatal outcomes between the anterior and posterior groups, except in the incidence of placenta accreta (Tables 3 and 4 ).

Table 3. Comparison between maternal characteristics in the anterior and the posterior groups.

\begin{tabular}{|c|c|c|c|c|c|c|c|c|c|}
\hline & \multicolumn{5}{|c|}{ Complete placenta previa } & \multicolumn{4}{|c|}{ Incomplete placenta previa } \\
\hline & Anterior & Posterior & OR & $(95 \% \mathrm{CI})$ & $P$ value & Anterior & Posterior & OR $\quad(95 \% \mathrm{CI})$ & \\
\hline & $\mathrm{n}=21$ & $\mathrm{n}=50$ & & & & $\mathrm{n}=10$ & $\mathrm{n}=81$ & & value \\
\hline Maternal age at delivery (years) & $34.0 \pm 3.9$ & $33.4 \pm 4.6$ & & & 0.578 & $34.0 \pm 5.0$ & $33.3 \pm 4.3$ & & 0.731 \\
\hline Nulligravida & $7(33.3 \%)$ & $16(32.0 \%)$ & 1.06 & $(0.36-3.14)$ & 0.866 & $3(30.0 \%)$ & $21(25.9 \%)$ & $1.22(0.29-5.17)$ & 0.917 \\
\hline Nullipara & $7(33.3 \%)$ & $22(44.0 \%)$ & 0.64 & $(0.22-1.85)$ & 0.569 & $3(30.0 \%)$ & $36(44.4 \%)$ & $0.54(0.13-2.22)$ & 0.595 \\
\hline Prior preterm delivery & $3(14.3 \%)$ & $1(2.0 \%)$ & 8.17 & $(0.80-83.67)$ & 0.138 & $0(0.0 \%)$ & $2(2.5 \%)$ & $(1-1)$ & 0.522 \\
\hline Prior cesarean delivery & $7(33.3 \%)$ & $6(12.0 \%)$ & 3.67 & $(1.06-12.74)$ & 0.074 & $1(10.0 \%)$ & $5(6.2 \%)$ & $1.69(0.18-16.11)$ & 0.83 \\
\hline Prior dilatation and curettage & $7(33.3 \%)$ & $17(34.0 \%)$ & 0.97 & $(0.33-2.86)$ & 0.825 & $2(20.0 \%)$ & $32(39.5 \%)$ & $0.38(0.08-1.92)$ & 0.392 \\
\hline ART & $1(4.8 \%)$ & $6(12.0 \%)$ & 0.37 & $(0.04-3.25)$ & 0.619 & $1(10.0 \%)$ & $5(6.2 \%)$ & $1.69(0.18-16.11)$ & 0.83 \\
\hline
\end{tabular}

Data are expressed as mean \pm SD or number $(\%)$. OR, odds ratio. CI, confidence interval.

Table 4. Comparison between perinatal outcomes in the anterior and the posterior groups.

\begin{tabular}{|c|c|c|c|c|c|c|c|c|c|c|}
\hline \multicolumn{6}{|c|}{ Complete placenta previa } & \multicolumn{5}{|c|}{ Incomplete placenta previa } \\
\hline & Anterior & Posterior & OR & $(95 \% \mathrm{CI})$ & P value & Anterior & Posterior & OR & $(95 \% \mathrm{CI})$ & \\
\hline & $\mathrm{n}=21$ & $\mathrm{n}=50$ & & & & $\mathrm{n}=10$ & $\mathrm{n}=81$ & & & value \\
\hline Admission & $16(76.2 \%)$ & $28(56.0 \%)$ & 2.51 & $(0.80-7.93)$ & 0.183 & $3(30.0 \%)$ & $23(28.4 \%)$ & 1.08 & $(0.26-4.54)$ & 0.791 \\
\hline Use of tocolytics & $12(57.1 \%)$ & $19(38.0 \%)$ & 2.18 & $(0.77-6.13)$ & 0.222 & $2(20.0 \%)$ & $10(12.3 \%)$ & 1.78 & $(0.33-9.57)$ & 0.857 \\
\hline $\begin{array}{l}\text { Antepartum } \\
\text { bleeding }\end{array}$ & $16(76.2 \%)$ & $27(54.0 \%)$ & 2.73 & $(0.86-8.59)$ & 0.139 & $2(20.0 \%)$ & $14(17.3 \%)$ & 1.2 & $(0.23-6.25)$ & 0.82 \\
\hline $\begin{array}{l}\text { Gestational age at } \\
\text { bleeding onset } \\
\text { (weeks) }\end{array}$ & $\begin{array}{l}26.4 \\
(17.1-36.7)\end{array}$ & $31.4(18.7-36.6)$ & & & 0.016 & $32.4(31.7-33.1)$ & $32.1(25.4-35.1)$ & & & 0.095 \\
\hline $\begin{array}{l}\text { Gestational age at } \\
\text { delivery (weeks) }\end{array}$ & $\begin{array}{l}36.7 \\
(25.6-37.7)\end{array}$ & $37.1(28.1-38.4)$ & & & $<0.001$ & $37.6(33.9-38.1)$ & $37.6(34.7-38.4)$ & & & 0.708 \\
\hline$<34$ weeks & $8(38.1 \%)$ & $5(10.0 \%)$ & 5.54 & $(1.55-19.85)$ & 0.014 & $1(10.0 \%)$ & $0(0.0 \%)$ & & NA & 0.21 \\
\hline$<37$ weeks & $16(76.2 \%)$ & $16(32.0 \%)$ & 6.8 & $(2.12-21.84)$ & 0.002 & $2(20.0 \%)$ & $6(7.4 \%)$ & 3.13 & $(0.54-18.14)$ & 0.462 \\
\hline Birth weight (g) & $\begin{array}{l}2364 \\
(610-3022)\end{array}$ & $2663(1290-3740)$ & & & 0.002 & $\begin{array}{l}2686 \\
(1958-3080)\end{array}$ & $2778(1962-3432)$ & & & 0.235 \\
\hline$<2000 \mathrm{~g}$ & $8(38.1 \%)$ & $4(8.0 \%)$ & 7.08 & $(1.84-27.27)$ & 0.006 & $1(10.0 \%)$ & $2(2.5 \%)$ & 4.39 & $(0.36-53.34)$ & 0.749 \\
\hline$<2500 \mathrm{~g}$ & $13(61.9 \%)$ & $15(30.0 \%)$ & 3.79 & $(1.30-11.04)$ & 0.025 & $3(30.0 \%)$ & $15(18.5 \%)$ & 1.89 & $(0.44-8.15)$ & 0.661 \\
\hline $\begin{array}{l}\text { Apgar Score } \\
1 \mathrm{~min}<7\end{array}$ & $5(23.8 \%)$ & $3(6.0 \%)$ & 4.9 & $(1.05-22.83)$ & 0.08 & $1(10.0 \%)$ & $2(2.5 \%)$ & 4.39 & $(0.36-53.34)$ & 0.749 \\
\hline $\begin{array}{l}\text { Apgar Score } \\
5 \mathrm{~min}<7\end{array}$ & $2(9.5 \%)$ & $1(2.0 \%)$ & 5.16 & $(0.44-60.27)$ & 0.428 & $0(0.0 \%)$ & $0(0.0 \%)$ & & NA & NA \\
\hline Umbilical arterial $\mathrm{pH}$ & $\begin{array}{l}7.330 \\
(7.118-7.493)\end{array}$ & $7.326(7.018-7.413)$ & & & 0.859 & $\begin{array}{l}7.318 \\
(7.228-7.366)\end{array}$ & $7.316(7.057-7.425)$ & & & 0.815 \\
\hline Placenta accreta & $6(28.6 \%)$ & $2(4.0 \%)$ & 9.6 & $(1.75-52.66)$ & 0.01 & $2(20.0 \%)$ & $0(0.0 \%)$ & & NA & 0.003 \\
\hline $\begin{array}{l}\text { Cervical length at } \\
\text { delivery } \leq 35 \mathrm{~mm}\end{array}$ & $10(47.6 \%)$ & $23(46.0 \%)$ & 1.07 & $(0.38-2.96)$ & 0.892 & $4(40.0 \%)$ & $32(39.5 \%)$ & 1.02 & $(0.27-3.90)$ & 0.755 \\
\hline $\begin{array}{l}\text { Intraoperative blood } \\
\text { loss (ml) }\end{array}$ & $\begin{array}{l}1850 \\
(540-12870)\end{array}$ & $1315(360-6720)$ & & & 0.037 & $\begin{array}{l}1190 \\
(350-6120)\end{array}$ & $1100(400-3500)$ & & & 0.819 \\
\hline
\end{tabular}




\section{Discussion}

Prematurity is a consistent finding in studies reporting on women with placenta previa $5,12,13$. However, clinical courses widely vary with each patient. Some patients necessitate preterm cesarean section and hysterectomy for life-threatening hemorrhage, whereas others undergo elective cesarean section at term without hemorrhagic complications.

In this study, antepartum hemorrhage and preterm delivery were significantly more prevalent in women with complete placenta previa. However, the association between preterm delivery risk and placenta previa type remains controversial. Dola et al. reported that premature delivery was more common in women with complete placenta previa ${ }^{7}$. Bahar et al. reported that antepartum hemorrhage in women with placenta previa, especially major placenta previa (complete and partial placenta previa), were associated with preterm delivery ${ }^{8}$. On the contrary, Tuzovic et al. reported no difference in the frequency of preterm delivery between women with complete and incomplete placenta previa ${ }^{6}$. Daskalakis et al. also reported no differences in gestational age at delivery between different placenta previa types ${ }^{5}$. The discrepancies between these studies may have resulted from differences in maternal background, gestational period at diagnosis, or patient management.

In this study, only $19.1 \%$ women exhibited anterior placental position. This low rate suggests that placental tissue preferentially develops on the posterior uterine wall in placenta previa. A previous study reported that the incidence of placental migration is higher and the migration rate is faster in women with anterior placenta previa ${ }^{14}$. Moreover, anterior placental position is reportedly related to multiparity and history of more than two cesarean sections ${ }^{9}$. However, we observed no significant differences in parity and prior cesarean delivery between the anterior and posterior groups, irrespective of the placenta previa type. This may be because of the relatively low parity of our subjects: only four patients were multiparous, and none had undergone more than two cesarean sections. On the other hand, the incidence of placenta accreta was significantly higher in the anterior group, irrespective of placenta previa type. Eight of ten patients with placenta accreta $(80 \%)$ were in the anterior group, and six of eight patients (75\%) with anterior placenta accreta had prior cesarean sections. This concurs with previous reports, which suggest that placenta accreta develops through placental implantation over a cesarean scar 9, 15,16.

The present study also revealed that gestational age at bleeding onset was significantly lower in the anterior group in women with complete placenta previa. Consequently, the incidences of preterm de- livery and low birth weight were significantly higher in the anterior group. Interestingly, in women with incomplete placenta previa, anterior placental position did not significantly influence perinatal outcomes such as bleeding onset and preterm delivery. These results suggest that anterior placental position confers a remarkable risk of early bleeding and preterm delivery only in women with complete placenta previa. Hasegawa et al., while analyzing the ultrasound parameters for emergency cesarean section in placenta previa, reported no statistically significant increased risk with anterior placental position ${ }^{17}$. This may because women with incomplete placenta previa constituted $36 \%$ of the study population in their analysis.

The reasons for prematurity in anterior placenta remain poorly understood. Previous studies have reported that a short cervical length is associated with preterm delivery not only in women with normal placental position but also in those with placenta previa 18,19 . In this study, although gestational age at delivery was significantly earlier in the anterior groups in complete placenta previa, no significant difference was observed in cervical length at delivery between the anterior and posterior groups. Therefore, complete placenta previa combined with anterior placental location may confer a higher risk of early cervical shortening than incomplete placenta previa or posterior placental location.

We speculated that mechanical stimulation of the anterior uterine wall during daily life is more frequent and direct than that of the posterior wall, which is protected by the pelvis. If the placenta is located on the anterior wall, such stimulation may cause uterine contractions and subsequent unknown reactions in the underlying decidua basalis, where abundant blood flow exists. However, a previous study revealed that background uterine electromyographic activity, measured from the abdominal surface in the middle trimester of pregnancy, was independent of placental implantation site ${ }^{20}$. Further study is necessary to explore the pathophysiology underlying the clinical differences associated with anterior and posterior placental position in women with complete placenta previa.

In conclusion, obstetricians should be aware of the high risk of preterm delivery for antepartum hemorrhage in women with complete placenta previa, particularly when the placenta is located on the anterior uterine wall. Conversely, women with incomplete placenta previa are at relatively low risk of preterm delivery, and the incidence of preterm delivery is not influenced by placental position.

\section{Competing Interests}

The authors have declared that no competing 


\section{interest exists.}

\section{References}

1. Cunningham FG, Leveno KJ, Bloom SL, et al. Obstetrical hemorrhage. In: Cunningham FG, Leveno KJ, Bloom SL, et al, eds. Williams Obstetrics, 23rd ed. New York: McGraw-Hill; 2009: 757-803.

2. Crane JM, Van den Hof MC, Dodds L et al. Maternal complications with placenta previa. Am J Perinatol. 2000; 17: 101-5.

3. Oyelese Y, Smulian JC. Placenta previa, placenta accreta, and vasa previa. Obstet Gynecol. 2006; 107: 927-41.

4. Salihu HM, Li Q, Rouse DJ et al. Placenta previa: neonatal death after live births in the United States. Am J Obstet Gynecol. 2003; 188: 1305-9

5. Daskalakis G, Simou M, Zacharakis D et al. Impact of placenta previa on obstetric outcome. Int J Gynaecol Obstet. 2011; 114: 238-41.

6. Tuzovic L. Complete versus incomplete placenta previa and obstetric outcome. Int J Gynaecol Obstet. 2006; 93: 110-7.

7. Dola CP, Garite TJ, Dowling DD et al. Placenta previa: does its type affect pregnancy outcome? Am J Perinatol. 2003; 20: 353-60.

8. Bahar A, Abusham A, Eskandar M et al. Risk factors and pregnancy outcome in different types of placenta previa. J Obstet Gynaecol Can. 2009; 31: 126-31.

9. Jang DG, We JS, Shin JU et al. Maternal outcomes according to placental position in placental previa. Int J Med Sci. 2011; 8: 439-44.

10. Jang DG, Jo YS, Lee SJ et al. Risk factors of neonatal anemia in placenta previa. Int J Med Sci. 2011; 8: 554-7.

11. Dashe JS, McIntire DD, Ramus RM et al. Persistence of placenta previa according to gestational age at ultrasound detection. Obstet Gynecol. 2002; 99: 692-7.

12. Ananth CV, Demissie K, Smulian JC et al. Relationship among placenta previa, fetal growth restriction, and preterm delivery: a population-based study. $\mathrm{Ob}-$ stet Gynecol. 2001; 98: 299-306.

13. Zlatnik MG, Cheng YW, Norton ME et al. Placenta previa and the risk of preterm delivery. J Matern Fetal Neonatal Med. 2007; 20: 719-23.

14. Cho JY, Lee $\mathrm{YH}$, Moon MH et al. Difference in migration of placenta according to the location and type of placenta previa. J Clin Ultrasound. 2008; 36: 79-84.

15. Miller DA, Chollet JA, Goodwin TM. Clinical risk factors for placenta previa-placenta accreta. Am J Obstet Gynecol. 1997; 177: 210-4.

16. Allahdin S, Voigt S, Htwe TT. Management of placenta praevia and accreta. J Obstet Gynecol. 2011; 31: 1-6.

17. Hasegawa J, Higashi M, Takahashi $\mathrm{S}$ et al. Can ultrasonography of the placenta previa predict antenatal bleeding? J Clin Ultrasound. 2011; 39: 458-62.

18. Ghi T, Contro E, Martina $\mathrm{T}$ et al. Cervical length and risk of antepartum bleeding in women with complete placenta previa. Ultrasound Obstet Gynecol. 2009; 33: 209-12.

19. Stafford IA, Dashe JS, Shivvers SA et al. Ultrasonographic cervical length and risk of hemorrhage in pregnancies with placenta previa. Obstet Gynecol. 2010; 116: 595-600.

20. Grgic O, Matijevic R, Vasilj O. Placental site does not change background uterine electromyographic activity in the middle trimester of pregnancy. Eur J Obstet Gynecol Reprod Biol. 2006; 127: 209-12. 